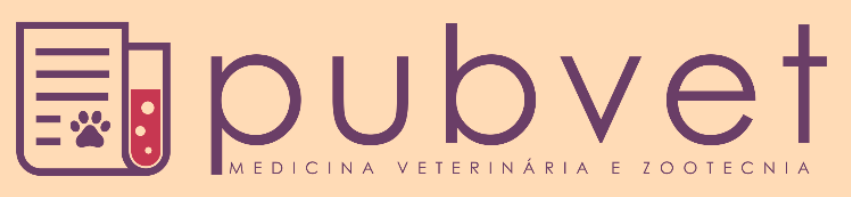

https://doi.org/10.31533/pubvet.v14n4a541.1-15

\title{
Biotécnicas investigativas para avaliação da glândula mamária dos animais de produção
}

\author{
Thaíse Cristine Ferreira de Carvalho-Sombra ${ }^{1} \bullet$, Diana Célia Sousa Nunes-Pinheiro ${ }^{2^{*}} \bullet$ \\ ${ }^{I}$ Doutoranda do Programa de Pós-Graduação em Ciências Veterinárias. Faculdade de Veterinária. Fortaleza-Ce Brasil. \\ ${ }^{2}$ Professora da Universidade Estadual do Ceará. Faculdade de Veterinária. Fortaleza-Ce. Brasil. \\ *Autor para correspondência, E-mail: diana.pinheiro@uece.br
}

\begin{abstract}
Resumo. A implementação de novas técnicas de avaliação da glândula mamária revela o diagnóstico e prognóstico das principais alterações que afetam o úbere, resultando, assim, em uma diminuição dos prejuízos econômicos dos produtores e um consequente aumento do bem-estar animal. As técnicas in vivo e in vitro auxiliam, individualmente ou em conjunto, a detecção precoce de alterações nas estruturas da glândula mamária nos principais animais de produção, por adicionar informações semiológicas pertinentes e necessárias na identificação do problema e sua consequente solução. Com enfoque na minimização das perdas de produção e qualidade de leite nos animais com alta produção, evitar distúrbios alimentares dos lactentes e morte por inanição, além de prevenir enfermidades da glândula mamária que corroboram com a inexistência do bem-estar desses animais. Dessa forma, a implementação de técnicas de investigação da glândula mamária abre novas perspectivas para estudos envolvendo o ambiente mamário em diversas perspectivas, a fim de diagnosticar e instituir a melhor terapêutica a ser utilizada.
\end{abstract}

Palavras chave: técnica in vitro, técnicas in vivo, úbere

\section{Investigative biotechniques for evaluation of the mammary gland of production animals}

\begin{abstract}
The implementation of new techniques to evaluate the mammary gland reveals the diagnosis and prognosis of the main changes affecting the udder, thus resulting in a reduction of producers' economic losses and a consequent increase in animal welfare. In vivo and in vitro techniques help, individually or jointly, the early detection of changes in mammary gland structures in the main production animals, by adding pertinent and necessary semiological information in the identification of the problem and its consequent solution. With a focus on minimizing milk yield and quality losses in high-yielding animals, avoiding infant feeding disorders and starvation death, and preventing mammary gland diseases that corroborate the lack of welfare of these animals. Thus, the implementation of mammary gland investigation techniques opens new perspectives for studies involving the mammary environment in different perspectives, in order to diagnose and institute the best therapy to be used.
\end{abstract}

Keywords: in vitro techniques, in vivo techniques; udder

\section{Biotecnologías de investigación para la evaluación de la glándula mamaria de animales de producción}

Resumen. La implementación de nuevas técnicas para evaluar la glándula mamaria revela el diagnóstico y el pronóstico de los principales cambios que afectan la ubre, lo que resulta 
en una reducción de las pérdidas económicas de los productores y el consiguiente aumento del bienestar animal. Las técnicas in vivo e in vitro ayudan, individual o conjuntamente, a la detección temprana de cambios en las estructuras de las glándulas mamarias en los principales animales de producción, al agregar información semiológica pertinente y necesaria en la identificación del problema y su consecuente solución. Con un enfoque en minimizar las pérdidas del rendimiento y calidad de la leche en animales de alta producción, evitar los trastornos alimentarios de los lactantes y la muerte por inanición, y prevenir enfermedades de las glándulas mamarias que corroboran con la falta de bienestar de estos animales. Por lo tanto, la implementación de técnicas de investigación de la glándula mamaria abre nuevas perspectivas para los estudios que involucran el entorno mamario en diferentes perspectivas, con el fin de diagnosticar e instituir la mejor terapia que se utilizará.

Palabras clave: técnica in vitro, técnicas in vivo, ubre

\section{Introdução}

A saúde da glândula mamária desempenha um papel importante na pecuária leiteira, sendo a base de um processo econômico da produção de leite e alvo de diversas alterações, tanto no parênquima glandular quanto no estroma. Essas modificações ocasionam a diminuição da produção e da qualidade do leite, elevando os custos com tratamentos, podendo ocasionar o abate precoce de vacas leiteiras. Neste contexto, a mastite é uma importante alteração patológica, ocasionada por distúrbios do fluxo de leite que interferem diretamente na saúde do úbere (Franz et al., 2009), sendo importante realizar periodicamente a avaliação da glândula mamária como forma de controle desta afecção. Na avaliação da glândula mamária, faz-se necessário realizar inicialmente o exame físico geral do animal, com posterior inspeção e palpação do órgão, seguido da avaliação macroscópica e microscópica do leite. Assim, a utilização de técnicas adicionais surge como auxílio para um diagnóstico e prognóstico rápido e precisos em animais com alterações na glândula mamária, facilitando assim o manejo e terapia adequada (Ibrahim et al., 2019). Portanto esse trabalho tem como objetivo realizar um levantamento das principais biotécnicas de investigação para a avaliação da glândula mamária dos animais de produção.

\section{Anatomofisiologia da glândula mamária}

A anatomia da glândula mamária difere nas diversas espécies domésticas. Entretanto, microscopicamente são bastante semelhantes (Hughes et al., 2018). Na vaca, a glândula mamária é agrupada em quatro, enquanto em outros ruminantes, como cabra e ovelha, é reunida em grupos de dois para formar o úbere. Este, por sua vez, é composto pelo ligamento suspensor mediano, o qual separa parcialmente as metades esquerda e direita, enquanto os quartos dianteiro e traseiro são separados por uma fina membrana. Não há compartilhamento dos sistemas de ductos do leite entre os quartos mamários (Nickerson \& Akers, 2011). O leite é secretado nos alvéolos, células secretoras esféricas e dispostas em uma única camada. Esses são circundados por células mioepiteliais ou musculares que se contraem para drenar o leite do lúmen alveolar para os ductos lactíferos. A união de vários alvéolos é denominada de lóbulos e os ductos que drenam os alvéolos convergem em um ducto comum, denominado de ducto intralobular. O conjunto de vários lóbulos forma um lobo, que forma o tecido glandular do órgão e é drenado por um ducto interlobular comum o qual o leite flui para a cisterna da glândula (Alhussien et al., 2016) (Figura 1).

O leite presente na cisterna da glândula flui distalmente para a cisterna do teto e em sua união há uma constrição conhecida como dobra anular. A cisterna do teto é a cavidade interna da papila que é revestida por um epitélio de dupla camada e apresenta numerosas dobras longitudinais e horizontais na mucosa. Distalmente encontra-se o canal do teto, a abertura pelo o qual o leite é escoado afluindo no orifício do teto. $\mathrm{Na}$ junção do canal com a cisterna do teto, estão presentes dobras longitudinais do revestimento que convergem para formar a roseta de Fuhrstenberg. O epitélio de dupla camada muda abruptamente distalmente para epitélio escamoso estratificado, que é contínuo com o revestimento externo do teto. A partir do orifício do teto emerge um músculo circular bem desenvolvido, formando o esfíncter do teto, cuja função é reter o leite nos intervalos entre a amamentação ou a ordenha (Nickerson \& Akers, 2011). 


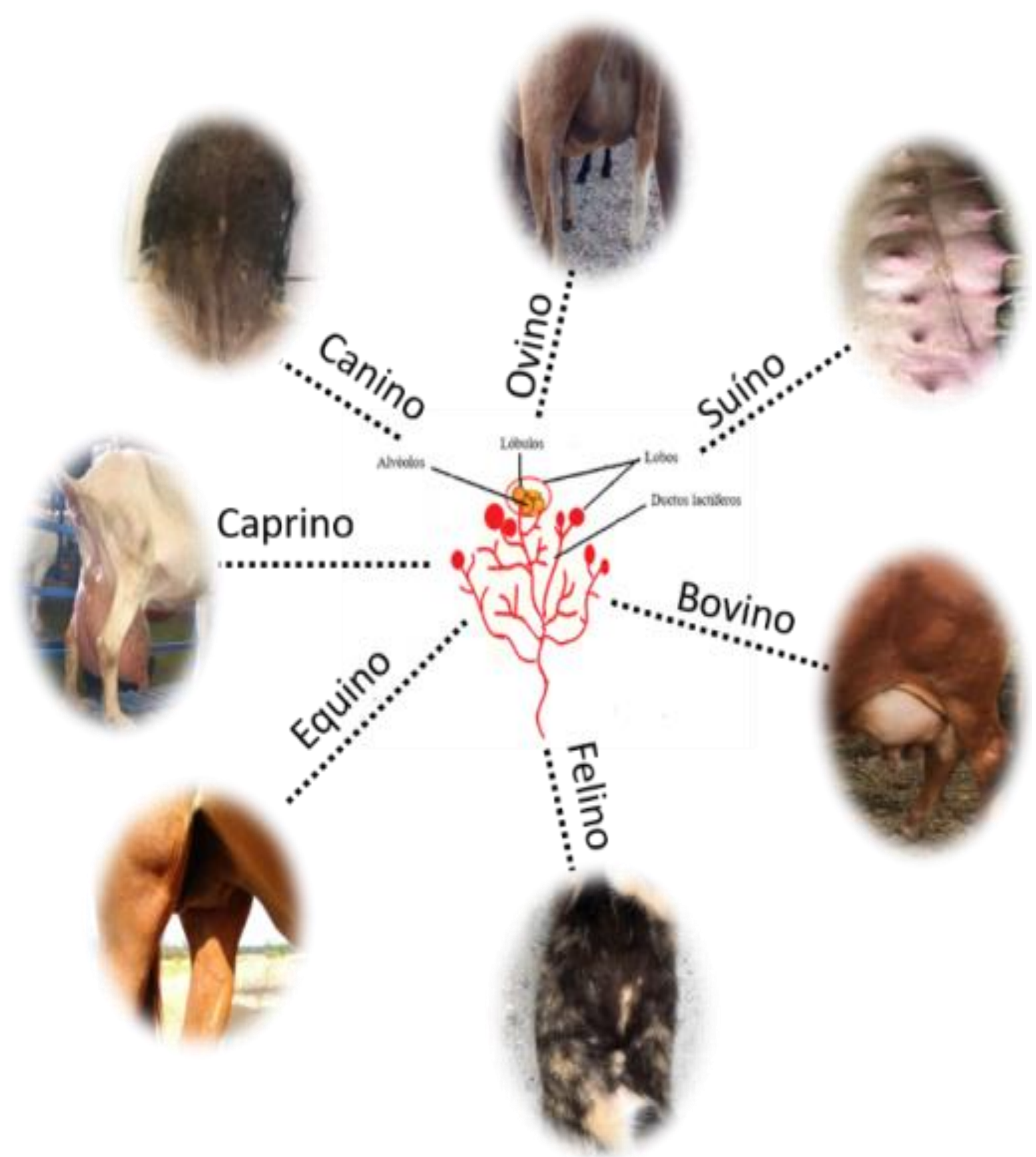

Figura 1. Glândula mamária dos animais e estruturas funcional do parênquima mamário.

\section{Técnicas de avaliação da glândula mamária}

São utilizadas várias técnicas na glândula mamária que auxiliam no diagnóstico presuntivo ou conclusivo de diferentes patologias mamárias nas várias espécies. Dentre essas técnicas destacam-se os métodos in vivo, que incluem a teloscopia, ultrassonografia, radiografia e a termografia infravermelho (Tabela 1), as técnicas in vitro tais como: citometria de fluxo, hibridização in situ, ELISA, citologia, histologia e MALDI-TOF (Tabela 2). Os métodos in vivo auxiliam na identificação e análise das características em tempo real dos constituintes mamários, além de serem de aplicação prática. Entretanto, podem ser influenciados pelo ambiente e pelos aspectos individuais do animal. Ao contrário, as técnicas in vitro, as análises são realizadas, em ambiente controlado. Todavia, necessitam de equipamentos, meios e reagentes específicos de alto custo. Assim, a aplicação dessas técnicas auxilia no aprofundamento dos conhecimentos de anatomia e fisiologia mamária, como também contribuem para identificação das patologias de forma representativa influindo no diagnóstico e tratamento preciso. 


\section{Técnicas in vivo}

\section{Teloscopia}

A endoscopia do teto ou teloscopia é utilizada para diagnosticar desordens na parte inferior da glândula mamária, avaliando principalmente as estruturas do canal do teto, cisterna do teto, roseta de Fuhrstenberg e cisterna da glândula. Além disso, esta técnica pode ser usada com propósitos curativos, no restabelecimento das lesões e estenose do teto em vacas leiteiras de alto rendimento (Geishauser et al., 2005). A técnica baseia-se na utilização de um endoscópio equipado com uma câmera de vídeo e, quando necessário, usa-se a pinça de biópsia para a colheita de amostras de tecido mamário (Fasulko, 2012). Quando comparada com outras técnicas, demonstra ser uma técnica rápida, menos invasiva, não traumática e livre de complicações, como hemorragias e mastite (Vangroenweghe et al., 2006). Entretanto, possui como principais desafios à necessidade da utilização de um equipamento específico, além de não conseguir visualizar estruturas superiores da glândula mamária. A teloscopia é recomendada para um diagnóstico rápido de lesões localizadas no interior do teto que alteram o fluxo de leite, com visualização direta da mucosa, permitindo inferências sobre a extensão e gravidade da lesão (Rathod et al., 2009). É comumente utilizada nas intervenções cirúrgicas em animais nos casos de estenose da teta (Franz, 2009) assim como para caracterizar a gravidade e extensão da lesão papilar além de identificar as alterações da passagem do leite entre as cisternas (Miranda et al., 2017) (Tabela 1).

Tabela 1. Técnicas in vivo utilizadas na medicina veterinária.

\begin{tabular}{|c|c|c|c|}
\hline Técnicas & Espécie & Indicação & Citações \\
\hline \multirow[t]{3}{*}{ Ultrassonografia } & Ruminantes & Avaliação mamária & 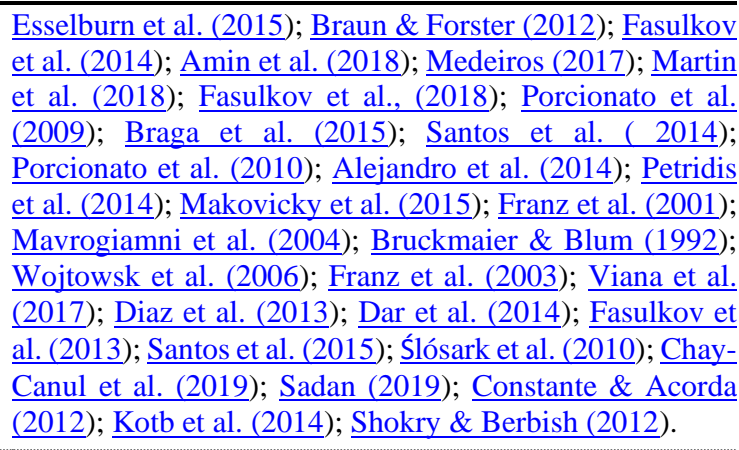 \\
\hline & \multirow[b]{2}{*}{ Bovinos } & Mastite & Porcinato et al. (2010); Risvanli et al. (2019). \\
\hline & & $\begin{array}{l}\text { Distúrbios no fluxo de leite e } \\
\text { alterações no teto }\end{array}$ & $\begin{array}{l}\text { Worstorff et al. (1986); Jenninger (1989); Takeda (1989); } \\
\text { Will et al (1990); Dreyfuss et al. (1990); Banting et al. } \\
\underline{\text { (1998); Saratsis \& Grunert (1993); Seeh et al. (1996). }}\end{array}$ \\
\hline Teloscopia & Bovinos & Avaliação mamária & $\underline{\text { See et al. (1996); Rathod et al. (2009) }}$ \\
\hline \multirow[t]{2}{*}{ Radiografia } & $\begin{array}{l}\text { Bovinos e } \\
\text { caprinos }\end{array}$ & Anatomia mamária & $\underline{\text { Bileck \& Janovsky (1956) }}$; Formston (1972); $\underline{\text { Adam et al. }}$ \\
\hline & Camelos & Anatomia mamária & $\underline{\text { Saleh et al. (1971). }}$. \\
\hline $\begin{array}{l}\text { Tomografia } \\
\text { computadorizada } \\
\text { /RM }\end{array}$ & Caprino & Avaliação mamária & Fowler et al. (1990); Gonzalez-Romano (2000) \\
\hline $\begin{array}{l}\text { Termografia } \\
\text { infravermelho }\end{array}$ & \multicolumn{2}{|c|}{ RuminanatesMastite } & $\begin{array}{l}\text { Rekant et al. (2015); Polat et al. (2010); Sathiyabarathi et } \\
\text { al. (2018); Hovinen et al. (2008); Metzener et al. (2014); } \\
\text { Metzener et al. (2015); Castro-Costa et al. (2014); Martins } \\
\text { et al. (2013); Nogueira et al. (2013); Chacur (2017); } \underline{\text { Sinha }} \\
\underline{\text { et al. (2019). }}\end{array}$ \\
\hline
\end{tabular}

Fonte: Elaborado pelos autores.

\section{Ultrassonografia}

A ultrassonografia (US) mamária é um método de diagnóstico por imagem, que fornece informações em tempo real sobre a arquitetura e características de órgãos, sendo capaz de identificar as condições fisiológicas e patológicas destes (Peixoto et al., 2010). A técnica se baseia na emissão de ondas sonoras que são refletidas (ecos) ao encontrar uma barreira (tecidos) e posteriormente captadas pelo aparelho que transformadas em 
imagem (Rodrigues \& Rodrigues, 2009). É uma ferramenta importante de avaliação clínica, pois visualiza tamanho, formato, contorno, textura e localização de órgãos, tecidos e vasos, podendo monitorar as funções destes, além de detectar a presença de massas anormais, como tumores (Mannion, 2006). O exame ultrassonográfico (US) apresenta diversos benefícios, sendo preciso, seguro, de fácil execução, de baixo custo, não invasivo, além de possibilitar a visualização de todas as estruturas do úbere (teto e parênquima glandular) em tempo real (Blond \& Buczinski, 2009). As primeiras referências da aplicação do US na glândula mamária animal de produção são recentes na literatura veterinária, com o primeiro relato científico de Caruolo \& Mochrie (1967), que utilizaram o US no modo A á 1Mz para avaliação do úbere em vacas. Posteriormente, Cartee et al. (1986) utilizaram em vacas o US no modo B para avaliação de distúrbios na secreção do leite e alterações mamárias. Em seguida, esta técnica vem sendo amplamente utilizada na medicina veterinária (Tabela 1$)$.

\section{Radiografia}

O uso da radiografia (RAD) na prática veterinária aumentou consideravelmente nos últimos anos, em decorrência dos avanços modernos da cirurgia ortopédica associada à necessidade de investigação mais completa dos casos. Inicialmente, a RAD foi utilizada na prática clínica veterinária de pequenos animais e posteriormente se tornou extremamente útil nas investigações de claudicação em equinos. Essa técnica geralmente é empregada para confirmar o diagnóstico clínico ou realizar o diagnóstico diferencial de diferentes alterações (Tavernor \& Vaughan, 1962). A radiologia na glândula mamária ou mamografia é realizada para avaliar as estruturas morfológicas do órgão. Essa técnica tem sido utilizada em vários estudos da anatomia da glândula mamária de várias espécies (Tabela 1), sendo realizada especialmente no teto para detectar precocemente obstruções congênitas e adquiridas com intuito de realizar a melhor abordagem terapêutica e ou cirúrgica (Naeini \& Gholami, 1999). No entanto, apresenta como principal limitação sobreposição de imagens limitando a avaliação completa das áreas afetadas, necessitando assim de técnicas mais modernas, como a tomografia computadorizada e a ressonância magnética permitindo então maior reconhecimento da anatomia e patologia para uma melhor escolha terapêutica (Crijns et al., 2017).

\section{Termografia infravermelho}

A termografia infravermelha (TI) é uma técnica não invasiva de diagnóstico precoce que vem sendo estudada como uma ferramenta para identificar processos fisiológicos e patológicos, além de inferir informações sobre o comportamento animal por meio do calor emitido relacionado ao fluxo sanguíneo (McCafferty, 2007). O uso da TI na produção animal é inovador, econômico, rápido, eficiente e fornece informações importantes sem a necessidade de contato físico com os animais (Salles et al., 2016). A principal limitação desta técnica é que a temperatura superficial do corpo também é influenciada pela radiação solar, umedecimento e evaporação, sendo necessária à sua mensuração precisa nos períodos noturnos ou em baixas irradiações solares (McCafferty, 2007). O alto custo dos equipamentos é outro fator limitante (Eddy et al., 2001). Essa técnica foi utilizada preliminarmente na medicina veterinária na década de 60 , com seu uso limitado a hospitais universitários e somente na década de 80 , a TI pode ser utilizada na prática de diagnóstico equino, principalmente na deteç̧ão precoce de laminite, fraturas por estresse, tendinite, além de desordens clínicas (Turner, 2001). O primeiro relato da utilização da TI na glândula mamária foi alegado por Kotrbacek \& Nau (1985), no qual avaliaram a temperatura da pele de porcas em lactação juntamente com a sua relação entre os lactantes. Em seguida, essa técnica tem sido utilizada com frequência na identificação de alterações em animais (Tabela 1).

\section{Técnicas in vitro}

\section{Citometria de fluxo}

A citometria de fluxo (CF) é um método pelo o qual as características físicas e químicas das células são mensuradas à medida que elas viajam em suspensão até um ponto de detecção (Conington et al., 2008). Embora a microscopia seja o método mais comum para contagem diferencial de células no leite, existem entraves que impedem uma maior eficiência por existir uma maior morosidade e uma baixa repetibilidade devido a avaliação subjetiva de um número limitado de células. Além disso, a CF mensura um número elevado de células em um curto período, determina a fase inflamatória do úbere antes da presença dos sinais 
clínicos de mastite, e analisa a eficácia da terapêutica utilizada (Koess \& Hamann, 2008). Entretanto, possui como principais desvantagens o custo elevado e a manutenção dos equipamentos, além da necessidade de operador treinado (Garcia-Cordero et al., 2010). Essa técnica foi utilizada pela primeira vez por Hageltorn \& Saad (1986) para identificação e diferenciação de células inflamatórias e, desde então vem sendo utilizada com diferentes finalidades na medicina veterinária (Tabela 2).

Tabela 2. Técnicas in vitro utilizadas na medicina veterinária.

\begin{tabular}{|c|c|c|c|}
\hline Técnicas & Espécie & Indicação & Citações \\
\hline \multirow{3}{*}{$\begin{array}{l}\text { Citometria } \\
\text { de fluxo }\end{array}$} & \multirow{2}{*}{ Ruminantes } & Monitorização do úbere & $\begin{array}{l}\text { Pillai et al. (2000); Redelman et al. (1988); Rivas et al. } \\
\text { (2001); Schwarz et al. (2011). }\end{array}$ \\
\hline & & Imunomarcação celular & 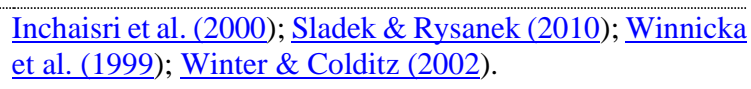 \\
\hline & Camelídeos & Imunomarcação celular & Al-Ashqar et al. (2015); Alluwaimi et al. (2017). \\
\hline \multirow{4}{*}{$\begin{array}{l}\text { Hibridizaçãc } \\
\text { in situ }\end{array}$} & \multirow[b]{2}{*}{ Bovinos } & $\begin{array}{l}\text { Detecção de patógenos no leite na } \\
\text { mastite }\end{array}$ & $\underline{\text { Gey et al. (2013). }}$. \\
\hline & & $\begin{array}{l}\text { Identificação e localização de RNAm } \\
\text { de peptídeos e proteínas }\end{array}$ & $\begin{array}{l}\text { Molenaar et al. (1996); Sayed-Ahmed et al. (2003); Bartha } \\
\text { et al. (2005). }\end{array}$ \\
\hline & \multirow{2}{*}{ Ovinos } & Mapa citogenético & Di Meo et al. (2007). \\
\hline & & Mastite & $\underline{\text { Pisanu et al. }(2015)}$. \\
\hline \multirow{2}{*}{ ELISA } & Ruminantes & Mastite & $\begin{array}{l}\text { Thielen et al. (2007); Chen et al. (2019); Leitner et al. } \\
\underline{\text { (2011); Sadiq et al. (2019); Winter \& Colditz (2002). }}\end{array}$ \\
\hline & Bovinos & $\begin{array}{l}\text { Imunomarcação de proteínas em } \\
\text { alterações mamárias }\end{array}$ & Zoldan et al. (2017); Soler et al.(2019) \\
\hline \multirow{4}{*}{ Citologia } & Bovinos & Avaliação morfológica mamária & Akers et al. (1981); Heald (1973). \\
\hline & Ruminantes & Mastite & $\begin{array}{l}\text { Giesecke \& Viljoen (1974); } \text { Nikerson \& Heald (1981); Da } \\
\text { Costa et al. (2004); Zhelavskyi et al. (2019); Varvil et al. } \\
\text { (2019); Chapaval et al. (2004). }\end{array}$ \\
\hline & $\begin{array}{l}\text { Pequenos } \\
\text { ruminantes }\end{array}$ & Avaliação da celularidade do leite & Bernacka et al. (2007); Lee \& Outteridge (1976). \\
\hline & Suínos & Avaliação da celularidade do leite & Nordin \& Lee (1982) \\
\hline \multirow{4}{*}{ Histologia } & \multirow{2}{*}{ Ruminantes } & $\begin{array}{l}\text { Avaliação morfométrica morfológica } \\
\text { mamária }\end{array}$ & 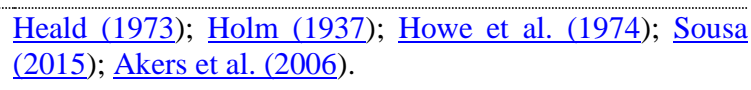 \\
\hline & & Mastite & $\begin{array}{l}\text { Thompson et al. (1978); Bianchi et al. (2019); Chang et al. } \\
\underline{(2019)} \text {; Fasulkov et al. (2015); Winter et al. (2004) }\end{array}$ \\
\hline & Camelídeos & $\begin{array}{l}\text { Avaliação morfométrica tecidual das } \\
\text { estruturas presente no úbere }\end{array}$ & Rizk et al. (2019); Kausra et al. (2001). \\
\hline & Caprinos & $\begin{array}{l}\text { Lesões papilomatosas cutâneas } \\
\text { mamárias virais }\end{array}$ & Manni et al. (1998). \\
\hline \multirow{2}{*}{\multicolumn{2}{|c|}{ MALDI-TOF Ruminantes }} & Mastite & $\begin{array}{l}\text { Magro et al. (2018); Katsafadou et al. (2019)} ; \underline{\text { Barreiro et }} \\
\underline{\text { al. (2010) }} ; \underline{\text { Boehmer et al. (2008) }} ; \underline{\text { Nonnemann et al. (2019)}} ; \\
\underline{\text { Rothen et al. (2019). }}\end{array}$ \\
\hline & & Detecção de fraudes no leite & Calvano et al. (2013). \\
\hline
\end{tabular}

Fonte: Elaborado pelos autores.

\section{Hibridização in situ}

A hibridização in situ (HInSitu) é uma técnica baseada na detecção de pequenos segmentos de RNA ou DNA oriundos de sequências de nucleotídeos específicos de material genético já conhecido. Dentre as várias técnicas disponíveis, a mais utilizada é a HInSitu por fluorescência que utiliza sondas oligonucleotídicas coradas com fluoróforo para marcar o RNA ribossômico (Gunasekera et al., 2003), e tem sido utilizada para detectar variações na expressão gênica entre os tecidos e as células (Molenaar et al., 1992). Essa técnica também pode ser aplicada na glândula mamária de várias espécies (Tabela 2). Essa ferramenta possui como principal vantagem a identificação rápida de microrganismos causadores da mastite. Uma das desvantagens é a falta de eficiência no diagnóstico de inflamações mamárias precoces, necessitando assim de outras técnicas auxiliares (Gey et al., 2013). 


\section{Elisa}

O ensaio imuno absorvente enzimático (ELISA) baseia-se na reação antígeno-anticorpo detectáveis por meio de reações enzimáticas e substrato, com resultado revelado pela mudança de coloração de uma substância denominada de cromógeno. Na rotina da medicina humana, o teste ELISA tem sido utilizado no diagnóstico de diversas patologias, com sua primeira aplicação sendo na identificação de cisticercose (Costa, 1986) e na doença de chagas (Rocha et al., 1987). Embora os testes sorológicos não possam ser considerados métodos de diagnóstico de primeira escolha, o teste ELISA apresenta algumas vantagens como a simplicidade, boa reprodutibilidade, alta sensibilidade e especificidade. É um teste frequentemente utilizado na medicina veterinária (Tabela 2). Entretanto, durante a evolução de algumas enfermidades de bovinos, a sensibilidade é limitada devido ao desenvolvimento tardio e irregular da resposta imune humoral (Ramos et al., 2015).

\section{Citologia}

A citologia é um método de avaliação celular na qual se observa as principais características morfológicas, de desenvolvimento e funções das estruturas celulares que compõem os tecidos e órgãos, permitindo a distinção entre processos inflamatórios agudos ou crônicos e neoplásicos (Chapaval et al., 2004). O estudo citológico mamário humano iniciou-se com a aspiração de células malignas por James Paget 1953 e desde então, a popularidade da técnica se difundiu devido ao baixo custo, à efetividade, à qualidade do procedimento, à rapidez diagnóstica e ao baixo índice de complicações, apresentando alta sensibilidade e especificidade. Dessa forma, a avaliação celular tem sido utilizada como ferramenta no diagnóstico de alterações em animais (Tabela 2). Contudo, essa técnica apresenta dificuldades na diferenciação de neoplasias, o que necessita de outras técnicas em conjunto para realizar o diagnóstico definitivo (Combalia, 2014). Dentre as técnicas citológicas mais utilizadas destacam-se a punção aspirativa por agulha fina (PAAF), a punção por capilaridade, os imprints e os raspados e escovados de diferentes secreções do organismo (Pinto, 2012).

\section{Histologia}

Histologia (HIS) é o ramo das ciências anatômicas que estuda tecidos de animais e plantas e as funções que exercem sobre o organismo (Gatner, 2017). A HIS normalmente é utilizada como técnica de diagnóstico confirmatório para diversas doenças, incluindo neoplasias (Murphy et al., 2004). Esse método além de visualizar as estruturas presentes no tecido, identifica padrões de infiltrados celulares, edema, congestão e alterações morfológicas celulares que facilitam o diagnóstico conclusivo e diferencial, auxiliando assim na implementação da melhor intervenção terapêutica (Heer et al., 2003). Assim, a utilização dessa técnica tem sido proposta em diferentes situações de diagnóstico na medicina veterinária (Tabela 2).

\section{Maldi-Tof}

A técnica de dessorção/ionização a laser assistida por matriz seguida de espectrometria de massas por tempo de voo (MALDI- TOF) possui aplicação na análise de rotina em todo mundo (Bizzini et al., 2010). É uma técnica multivalente no estudo de amostras biológicas, especialmente proteínas e peptídeos com a identificação dos pesos moleculares facilitando o rastreamento de moléculas em tecidos e células (Caprioli et al., 1997), além de ser um método alternativo para superar os erros da cultura microbiológica convencional (Braga et al., 2018). Foi introduzida como um método de ionização em grandes moléculas na década de 80 e desde então vem sendo difundida na análise proteômica (Vorm et al., 1994), revolucionando o campo da triagem bacteriana por ser um método muito mais rápido, confiável e altamente sensível para identificação desses microrganismos (Calvano et al., 2012). Essa técnica é utilizada na medicina veterinária para investigar alterações principalmente em ruminantes (Tabela 2).

\section{Considerações finais}

O estudo e aprofundamento das técnicas in vivo e in vitro mencionadas no presente trabalho auxiliam na análise semiológica da glândula mamária, pois investigam a anatomia e fisiologia do órgão, além de determinar suas possíveis alterações. Constituem, assim, ferramentas válidas para o diagnóstico das principais enfermidades que acometem a glândula mamária dos animais de produção, contribuindo com a escolha e a administração da terapêutica adequada, além de uma melhor qualidade do leite na produção animal. 


\section{Referências bibliográficas}

Adam, Z. A., Ragab, G. A., Awaad, A. S., Tawfiek, M. G., Abdel Maksoud, M. K. M. (2016). Anatomical and radiographical studies on the arterial supply of the udder in goat and their surgical importance. Beni-Suef University Journal of Basic and Applied Sciences,

Akers, R. A. M., Bauman, D. E., Goodman, G. T., Capuco, A. V., Tucker, A. H. (1981). Prolactin Regulation of Cytological Differentiation of Mammary Epithelial Cells in Periparturient Cows. Endocrinology, 109 (1), 31-40.

Akers, R. M., Capuco, A. V., Keys, J. E. (2006). Mammary histology and alveolar cell differentiation during late gestation and early lactation in mammary tissue of beef and dairy heifers. Livestock Science, 105, 44-49.

Al-Ashqar, R. A., Salem, K. A., Al Herz, A. K. M., Al-Haroon, A. I., Alluwaimi, A. M. (2015). The CD markers of camel (Camelus dromedarius) milk cells during mastitis: The LPAM-1 expression is an indication of possible mucosal nature of the cellular trafficking. Research in Veterinary Science, 99,77-81.

Alejandro, M., Rodríguez, M., Peris, C., Díaz, J. R. (2014). Study of ultrasound scanning as method to estimate changes in teat thickness due to machine milking in Manchega ewes. Small Ruminant Research, 119,138-145.

Alhussien, M., Manjari, P., Mohammed, S., Sheikh A.A., Reddi S., Dixit S. \& Dang A.K. (2016). Incidence of mastitis and activity of milk neutrophils in Tharparkar cows reared under semi-arid conditions. Tropical Animal Health and Production, 48 (6), 1291-1295.

Alluwaimi, A. M., Salem, K. T. A., Roqya A Al-Ashqer, R. A., Al-Shubaith, I. H. (2017). The Camel's (Camelus Dromedarius) Mammary Gland Immune System in Health and Disease. Journal of Advances of Dairy Research, 5, 1.

Amin, N. R., Patil, D. B., Kelawala, D. N., Parikh, P. V., Mer, D. R., Gameti, K. S., Gohil, K. M. (2018). Ultrasonography of udder and teat in dairy animals. Ruminant Science, 6 (1), 171-175.

Banting, A. (1998). Ultrasonographic examination of the mammary gland in cows with induced S. aureus mastitis: a criterion for prognosis and evaluation of therapy. Cattle Practice, v.6, p. 121-4.

Barreiro, J. R., Ferreira; C. R., Sanvido, G. B., Kostrzewa, M., Maier, T., Wegemann, B., Böttcher, V., Eberlin, M. N., Dos Santos, M. V. (2010). Short communication: Identification of subclinical cow mastitis pathogens in milk by matrix-assisted laser desorption/ionization time-of-flight mass spectrometry. Journal of Dairy Science, 93, 5661-5667.

Bartha, T., Sayed-Ahmeda, A., Rudas, P. (2005). Expression of leptin and its receptors in various tissues of ruminants. Domestic Animal Endocrinology, 29, 193-202.

Bernacka, H. (2006). Cytological quality of goat milk on the basis of the somatic cell count. Journal of Central European Agriculture, 7(4), 773-777.

Bianchi, R. M., Schwertz, C. I., De Cecco, B., Panziera, W., De Lorenzo, C., Heck, L. C., Snel, G. G. M., Lopes, B. C., Silva, F., Pavarini, S. P., Driemeier, D. (2019).Pathological and microbiological characterization of mastitis in dairy cows. Tropical Animal Health and Production, 51(7), 2057-2066.

Bileck, J. \& Janovsky, M. A. (1956). Radiographic Method for the Study of the function of the cow's udder. Nature, v.177, 582-583.

Bizzini, A., Durussel, C; Bille, J., Greub, G., Prod'hom, G. (2010). Performance of matrix-assisted laser desorption ionization-time of flight mass spectrometry for identification of bacterial strains routinely isolated in a clinical microbiology laboratory. Journal of Clinical Microbiology, 48 (5), 1549-1554.

Blond, L., Buczinski, S. (2009). Basis of ultrasound imaging and the main artifacts in bovine medicine. Veterinary Clinic and Food Animal, 25, 553-565.

Boehmer, J. L., Bannerman, D. D., Shefcheck, K., Ward, J. L. (2008). Proteomic Analysis of Differentially Expressed Proteins in Bovine Milk During Experimentally Induced Escherichia coli Mastitis. Journal of Dairy Science, 91, 4206-4218.

Braga, P. A. C., Gonçalves, J. L., Barreiro, J. R., Ferreira, C. R., Tomazi, T., Eberlin, M. N., Santos, M. V. (2018). Rapid identification of bovine mastitis pathogens by MALDI-TOF Mass Spectrometry. Pesquisa Veterinária Brasileira, 38 (4), 586-594. 
Braga, R. A., Tiron, S. M. T., Taira, A. R., Oliveira, M. G., Bonato, D. V., Vrisman, D. P., Silva, M. H., Teixeira, P. P. M. (2015). Morfofisiologia, afecções e diagnóstico ultrassonográfico da glândula mamária em bovinos: revisão de literatura. Nucleus Animalium, 7 (1), 17-30.

Braun, U., Forster, E. (2012). B-mode and colour Doppler sonographic examination of the milk vein and musculophrenic vein in dry cows and cows with a milk yield of 10 and $20 \mathrm{~kg}$. Acta Veterinaria Scandinavica, 54 (1):1-5.

Bruckmaier, R. M., Blum, J. W. (1992). B-mode ultrasonography of mammary glands of cows, goats and sheep during $\alpha$ - and / $\beta$ - adrenergic agonist and oxytocin administration. Journal of Dairy Research, 59:151-15.

Calvano, C. D., De Ceglie, C., Aresta, A., \& Facchini, L. A., Zambonin, C. G. (2013). MALDI-TOF mass spectrometric determination of intact phospholipids as markers of illegal bovine milk adulteration of high-quality milk. Anal Bioanal Chem, 405:1641-1649.

Calvano, C. D., De Ceglie, C., Monopolia, A., Zambonin, C. G. (2012). Detection of sheep and goat milk adulterations by direct MALDI-TOF MS analysis of milk tryptic digests. Journal Mass Spectrometry, 47:1141-1149.

Caprioli R. M., Farmer, T. B. \& Gile, J. (1997). Molecular Imaging of Biological Samples: Localization of Peptides and Proteins Using MALDI-TOF MS. Anal Chem, 69, 4751-4760.

Cartee Re., Ibrahim Ak., Mc Leary D. (1986). B-mode ultrasonography of the bovine udder and teat. $J$ American Veterinary Medical Association, 188 (11):1284-7.

Caruolo Ev., Mochrie Rd. (1967). Ultrasonograms of lactating mammary glands. Journal of Dairy Science, 50(2):225-30.

Castro-Costa, A., Caja, G., Salama, A. A. K., Rovai, M., Flores, C., Aguiló, J. (2014). Thermographic variation of the udder of dairy ewes in early lactation and following an Escherichia coli endotoxin intramammary challenge in late lactation. Journal of Dairy Science, 97:1377-1387.

Chacur, M. G. M. (2017). Termografia por infravermelho na reprodução de bubalinos. Revista Brasileira de Reprodução Animal, 41(1):180-187.

Chang, J., Yu, D., Chen, J., Wang, J., Wang, F., Jiang, Z. Z., He, X., Wu, R., Yu, L. (2019). Mycoplasma leachii causes bovine mastitis: Evidence from clinical symptoms, histopathology and immunohistochemistry. Journal of Integrative Agriculture, 18(1):160-168.

Chapaval, L., Oliveira, A. A. F., Santos, S. F. (2004). Avaliação da citologia de expressão no diagnóstico da mastite bovina e sua relação com o "california mastitis test" (cmt). 41 $1^{\text {a }}$ Reunião Anual da Sociedade Brasileira de Zootecnia - Campo Grande, MS.

Chay-Canul, A. J., Garcia-Herrera, R. A., Ojeda-Robertos, N. F., Macias-Cru, U., Vicente-Pérez, R., Meza-Villalvazo, V. M. (2019). Relationship between body condition score and subcutaneous fat and muscle area measured by ultrasound in Pelibuey ewes. Emirates Journal of Food and Agriculture, 31(1):53-58.

Chen, Z., Xu, X., Tan T., Chen, D., Liang, H., Sun, K., Li, M., Zhang, H., Mao, Y., Yang, Z. (2019). MicroRNA-145 regulates immune cytokines via targeting FSCNI in Staphylococcus aureus-induced mastitis in dairy cows. Reproduction Domestic Animal, 54, 882-891.

Combalia, N. (2014). Diagnóstico citológico en patología mamaria. Revista de Senología y Patología Mamaria, 27(4):183-189.

Conington, J., Cao, G., Stott, A., Bünger, L. (2008). Breeding for resistance to mastitis in United Kingdom sheep, a review and economic appraisal. Veterinary Record, 162:369-376.

Constante, J. L., Acorda, J. A. (2012). Ultrasound features of the udder and teat of water buffaloes (Bubalus bubalis L.) at different stages of lactation. Journal of Veterinary. Medicine, 49(2):76-82.

Costa, J. M. (1986). Teste imunoenzimático (elisa) no diagnóstico da neurocisticercose. Arquivo NeuroPsiquiatria, 44 (1):15- 31.

Crijns, C. P., Weller, R., Vlaminck, L., Verschooten, F., Schauvliege, S., Powell, S. E., Van Bree, H. J. J., Gielen, I. M. V. L. (2017). Comparison between radiography and computed tomography for diagnosis of equine skull fractures. Equine Veterinary Education, 31(10):543-550. 
Da Costa, E. O., Ribeiro, M. G., Ribeiro, A. R., Rocha, N. S., Nardi J'Unior, G. (2004). Diagnosis of clinical bovine mastitis by fine needle aspiration followed by staining and scanning electron microscopy in a Prototheca zopfii outbreak. Mycopathologia, 158:81-85.

Dar, M. U. D., Tiwari, D. K., Jhala, S. K., Patil, D. B., Parikh, P. V; Joy, N., Wadhwani, K. N. (2014). Ultrasonography of teat in surti goats. Indian Journal of Animal Research, 48(1):59-62.

Di Meo, G. P., Perucatti, A., Floriot, S., Hayes, H., Schibler, L., Rullo, R., Incarnato, D., Ferretti, L., Cockett, N., Cribiu, E., Williams, J. L., Eggen, A., Iannuzzi, L. (2007). An advanced sheep (Ovis aries, $2 \mathrm{n} 1 / 4$ 54) cytogenetic map and assignment of 88 new autosomal loci by fluorescence in situ hybridization. Animal Genetics, 38:233-240.

Díaz, J. R., Alejandro, M., Peris, C., Fernández, N. (2013). Use of ultrasound scanning to estimate teat wall thickness in Murciano-granadina goats. Livestock Science, 155:114-122.

Dreyfuss, D. J., Madison, J. B., Reef, V. B. (1990). Surgical treatment of a mural teat abscess in a cow. Journal of American Veterinary Medicine Association, 197(12):1629-1630.

Eddy, A. L., Van Hoogmoed, L. M., Snyder, J. R. (2001). The role of thermography in the management of equine lameness. The Veterinary Journal, 162:172-181.

Esselburn, K. M., Hill, T. M., Bateman Ii, H. G., Fluharty, F. L., Moeller, S. J., O'diam, K. M., Daniels K. M. (2015). Examination of weekly mammary parenchymal area by ultrasound, mammary mass, and composition in Holstein heifers reared on 1 of 3 diets from birth to 2 months of age. Journal of Dairy Science, 98:5280-5293.

Fasulkov, I. R. (2012). Ultrasonography of the mammary gland in ruminants: A review, Bulgarian Journal of Veterinary Medicine, 15(1):1-12.

Fasulkov, I., Karadaev, M., Vasilev, N., Nikolov, M., Nonov, T. (2018). Three-dimensional ultrasonography of the mammary gland in lactating cows. Tradition and Modernity in Veterinary Medicine, 3(5):109-113.

Fasulkov, I., Karadaev, M., Vasilev, N., Radostin Simeonov, R., Urumova, V., Mladenov, E. (2015). Ultrasound and histopathological investigations of experimentally induced Staphylococcus aureus mastitis in goats. Small Ruminant Research, 129:114-120.

Fasulkov, I., Vasilev, N., Karadaev, M., Dineva, G. (2014). Visualization and measurement of teat structures in black-and-white cows through ultrasonography. Mac Veterinary Review, 37(1):89-93.

Fasulkov, I., Yotov, S., Atanasov, A., Antonov, A. (2013). Evaluation of different techniques of teat ultrasonography in goats. Istanbul Üniv. Vet. Fak. Derg, 39(1):33-39.

Formston, C. (1972). Radiography Relative to the Mammary Gland and Teat of the Cow. Acta Radiologica: Diagnosis, 12(319):83-89.

Fowler, P. A., Knight, C. H., Cameron, G. G., Foster, M. A. (1990). Use of magnetic resonance imaging in the study of goat mammary glands in vivo. Journal of Reproduction and Fertility, 89:59-366.

Franz, S., Floek, M., Hofmann-Parisot, M. (2009). Ultrasonography of the Bovine Udder and Teat. Vet Clinical Food Animal, 25:669-685.

Franz, S., Hofmann-Parisot, M., Baumgartner, W., Windischbauer, G., Suchy, A., Bauder, B. (2001). Ultrasonography of the teat canal in cows and sheep. Veterinary Record, 49:109-112.

Franz, S., Hofmann-Parisot, M., Gütler, S., Baumgartner, W. (2003). Clinical and ultrasonographic findings in the mammary gland of sheep. New Zealand Veterinary Journal, 51(5):238-243.

Garcia-Cordero, J. L., Barrett, L. M., O’Kennedy, R., Ricco, A. J. (2010). Microfluidic sedimentation cytometer for milk quality and bovine mastitis monitoring. Biomed Microdevices, 12:1051-1059.

Gatner, L. P. (2017). Textbook of Histology. $4^{\mathrm{a}}$ edição. Philadelphia: Elsevier.

Geishauser, T., Vet, D. M., Habil, D. M. V., Fta., Msca., Querengasser, K Vetb, D.M., Querengasser, J. (2005). Teat Endoscopy (Theloscopy) for Diagnosis and Therapy of Milk Flow Disorders in Dairy Cows, Veterinary Clinic and Food Animal, 21:205-225.

Gey, A., Werckenthin, C., Poppert, S., Straubinger, R. K. (2013). Identification of pathogens in mastitis milk samples with fluorescent in situ hybridization. Journal of Veterinary Diagnostic Investigation, 25(3):386-394. 
Giesecke, W. H., Viljoen M. H. (1974). The diagnosis of subclinical mastitis in lactating cows: a comparison of cytological methods and a monovalent radial immunodiffusion test. Onderstepoort Journal Veternary Research, 41(2):51-74.

Gonzalez-Romano, N., Arencibia, A., Los Monteros, A. E., Rodriguez, E., Rivero, M., Vázquez, J. M., Capote, J., Jaber, J. R. (2001). Anatomical Evaluation of the Caprine Mammary Gland by Computed Tomography Radiology and Histology. Anatomy Histology and Embryology, 18:25-30.

Gunasekera, T. S., Dorsch, M. R., Slade, M. B., Veal, D.A. (2003). Specific detection of Pseudomonas spp. in milk by fluorescence in situ hybridization using ribosomal RNA directed probes. Journal of Applied Microbiology, 94:936-945.

Hageltorn, M., Saad, M. A. (1986). Flow cytofluorometric characterization of bovine blood and milk leukocytes. American Journal Veterinary Research, 47:2012-2116.

Heald, C. W. (1973). Hormonal Effects on Mammary Cytology. Journal of Dairy Science, 57 (8):917-925.

Heer, R., Shrimankar, J., Griffit, C. D. M. (2003). Granulomatous mastitis can mimic breast cancer on clinical, radiological or cytological examination: a cautionary tale. The Breast, 12:283-286.

Holm, G. C. (1937). A histological study of bovine udder parenchyma. Veterinary Medicine,32 (4), 163-167

Hovinen, M., Siivonen, J., Taponen, S., Hanninen, L., Pastell, N., Aisla, Am., Pyorala, S. (2008). Detection of clinical mastitis with help of a thermal camera. Journal of Dairy Science, 91(12):4580-4598.

Howe, J. E., Heald, C. W., Bibb, T. L. (1974). Histology of Induced Bovine Lactogenesis. Journal of Dairy Science, 58(6):853-860.

Hughes, K., Watson, C. J. (2018). The Mammary Microenvironment in Mastitis in Humans, Dairy Ruminants, Rabbits and Rodents: A One Health Focus. Journal of Mammary Gland Biology and Neoplasia, 23, 27-41.

Ibrahim, A. A. H., A. Adam, Z. E. A., Tawfiek, M. G. (2019). Cross sectional anatomy, magnetic resonance imaging and computed tomography of fetlock joint in camel. Journal of Veterinary Medical Research, 26(2):1-13.

Inchaisri, C., Persson Waller, K., Johannisson, A. (2000). Studies on the Modulation of Leucocyte Subpopulations and Immunoglobulins following Intramammary Infusion of b1,3- glucan into the Bovine Udder during the Dry Period. Journal of Veterinary Medicine, 47(5):373-386.

Jenninger, S. (1989). Ultrasonography of the bovine udder-physiological and pathological findings. Thesis; Munchen.

Katsafadou, A. I., Tsangaris, G. T., Anagnostopoulos, A. K., Charalambos Billinis, C., Mariana S. Barbagianni, M. S., Vasileiou, N. G. C., Spanos, S. A., Mavrogianni, V. S., Fthenakis, G. C. (2019). Proteomics data of ovine mastitis associated with Mannheimia haemolytica. Data in Brief, 25:1042-1059.

Kausra, R., Sarwar, A., Hayat, C. S. (2001). Gross and microscopic anatomy of mammary glands of dromedaries under different physiological conditions. Pakistan Veterinary, 21(4):189-193.

Koess, C., Hamann, J. (2008). Detection of mastitis in the bovine mammary gland by flow cytometry at early stages. Journal of Dairy Research, 75:225-232.

Kotb, E. E. Z., Abu-Seida, A. M., Fadel, M. S. (2014). The correlation between ultrasonographic and laboratory findings of mastitis in buffaloes. Global Veterinaria, 13(1):68-74.

Kotrbacek, V., Nau, H. R. (1984). The changes in skin temperatures of periparturient sows. Acta Veterinary Brno:54, 35-40.

Lee, C. S., Outteridge, P. M. (1976). The identification and ultrastructure of macrophages from the mammary gland of the ewe. Ajebak, 54(1):43-55.

Leitner, G., Sapeiroa, S., Krifucks, O., Weisblit, L., Lavi, Y., Heller, E. D. (2011). Systemic and local mammary gland immunity to udder infection in goats by various Staphylococcus species. Small Ruminant Research, 95:160-167.

Magro, M., Zaccarin, M., Miotto, G., Da Dalt, L., Baratella, D., Fariselli1, P., Gabai, G., Vianello, F. (2018). Analysis of hard protein corona composition on selective iron oxide nanoparticles by MALDI-TOF mass spectrometry: identification and amplification of a hidden mastitis biomarker in milk proteome. Analytical and Bioanalytical Chemistry, 410(12):2949-2959. 
Makovický, P., Margetín, M., Milerski, M. (2015). Estimation of udder cistern size in dairy ewes by ultrasonography. Mljekarstvo, 65(3):210-218.

Manni, V., Roperto, F., Di Guardo, G., Galati, D., Condoleo, R. U. \& Venuti, A. (1998). Presence of papillomavirus-like DNA sequences in cutaneous fibropapillomas of the goat udder. Veterinary Microbiology, 61, 1-6.

Mannion, P. (2006). Diagnostic ultrasound in small animal practice. Blackwell Science: Oxford.

Martin, L. M., Stöcker, C., Sauerwein, H., Büscher, W., Müller, U. (2018). Evaluation of inner teat morphology by using high-resolution ultrasound: Changes due to milking and establishment of measurement traits of the distal teat canal. Journal of Dairy Science, 101:1-12.

Martins, R. F. S., Paim, T. P., Cardoso, C. A., Dallago, B. S. L., Melo, C. B., Louvandini, H., Mcmanus, C. (2013). Mastitis detection in sheep by infrared thermography. Research in Veterinary Science, 94:722-724.

Mavrogianni, V. S., Fthenakis, G. C., Burriel, A. R., Gouletsou, P., Papaioannou, N., Taitzoglou, I. A. (2004). Experimentally Induced Teat Stenosis in Dairy Ewes: Clinical, Pathological and Ultrasonographic Features. Journal of Compendium Pathology, 130:70-74.

Mccafferty, D. J. (2007). The value of infrared thermography for research on mammals: previous applications and future directions. Mammal Review, 37(3):207-223.

Medeiros, B. P. (2017). Caracterização ultrassonográfica e histopatológica de lesões de glândulas mamárias de vacas de descarte. Dissertação, Programa de Pós-Graduação em Medicina Veterinária -Patologia e Ciências Clínicas, Seropédica-RJ.

Metzner, M., Sauter-Louis, C., Seemueller, A. Petzl, W. \& Zerbe, H. (2015). Infrared thermography of the udder after experimentally induced Escherichia coli mastitis in cows. The Veterinary Journal, 204, 360-362.

Metzner, M., Sauter-Louis, C., Seemueller, A., Petzl, W. \& Klee, W. (2014). Infrared thermography of the udder surface of dairy cattle: Characteristics, methods, and correlation with rectal temperature Moritz. The Veterinary Journal, 199, 57-62.

Miranda, F. G., Cabala, R. W., Lima, J. A. M., Nepomuceno, A. C., Tôrres, R. C. S., Gheller, V. A. (2017). Ultrasonography and theloscopy for the diagnosis of obstructive fibrosis in the Fürstenberg venous ring in the four quarters of the udder of a cow: a case report. Arquivo Brasileiro de Medicina Veterinária e Zootecnia, 69(5):1125-1129.

Molenaar, A. J., Davis, S. R., Wilkins, R. J. (1992). Expression of a -Lactalbumin, a- S 1- Casein, and Lact oferrin Genes Is Heterogeneous in Sheep and Cattle Mammary Tissue. The Journal of Histochemistry and Cytochemistry, 40(5):611-618.

Molenaar, A. J., Kuys, Y. M., Davis, S. R., Wilkins, R. J., Mead, P. E., Tweedie, J. W. (1996). Elevation of Lactoferrin Gene Expression in Developing, Ductal, Resting, and Regressing Parenchymal Epithelium of the Ruminant Mammary Gland. Journal of Dairy Science, 79:1198-120.

Murphy, S., Sparks, A. H., Brearley, M. J., Smith, K. C., Blunden, A. S. (2004). Relationships between the histological grade of cutaneous mast cell tumours in dogs, their survival and the efficacy of surgical resection. Veterinary Record, 154:743-746.

Naeini, T. A., Gholami, A. (1999). Radiography of the Bovine Mammary Gland. Journal of Applied Animal Research, 15 (1), 53-56.

Nickerson, S. C., Akers, R. M. (2011). MAMMARY GLAND Anatomy. In: _. Encyclopedia of Dairy Sciences, 2. ed. [S.1.]: Academica Express.

Nogueira, F. R. B., Souza, B. B., Carvalho, M. G. X., Garino, J. F., Marques, A. V. M. S. (2013). Termografia infravermelha: uma ferramenta para auxiliar no diagnóstico e prognóstico de mastite em ovelha. Revista Brasileira de Medicina Veterinária, 35(3):289- 297.

Nonnemann, B., Lyhs, U., Svennesen, L., Kristensen, K. A., Klaas, I. C., Pedersen, K. (2019). Bovine mastitis bacteria resolved by MALDI-TOF mass spectrometry. Journal of Dairy Science, 102:1-10.

Nordin, W.C., Lee, S. (1982). Cytology of Milk in Guinea Pigs. Acta Anatomy, 113:135-144.

Peixoto, G. C. X., Lira, R. A., Alves, N. D. A., Silva, A.R. (2010). Bases Físicas da Formação da Imagem Ultrassonográfica. Acta Veterinaria Brasilica, 4 (1):15-24. 
Petridis, I. G., Gouletsou, P. G., Barbagianni, M. S., Amiridis, G. S., Christos Brozos, C., Valasi, I., Fthenakis, G. C. (2014). Ultrasonographic findings in the ovine udder during involution. Journal of Dairy Research, 81:288-296.

Pillai, S. R., Kunze, E., Sordillo, L. M., Jayarao, B. M. (2001). Application of Differential Inflammatory Cell Count as a Tool to Monitor Udder Health. Journal of Dairy Science, 84:1413-1420.

Pinto, F. R. G. (2012). Caderno de referência 2: citopatologia não ginecológica. CEPESC Brasília: Ministério da Saúde; Rio de Janeiro.

Pisanu, S., Cubeddu, T., Pagnozzi, D., Rocca, S., Cacciotto, C., Alberti, A., Marogna, G., Uzzau, S., Addis, M. F. (2015). Neutrophil extracellular traps in sheep mastitis. Veterinary Research, 46:59- 72.

Polat, B., Colak, A., Cengiz, M., Yanmaz, L. E., Oral, H., Bastan, A., Kaya, S., Hayirli, A. (2010). Sensitivity and specificity of infrared thermography in detection of subclinical mastitis in dairy cows. Journal of Dairy Science, 93(8):3524-3532.

Porcionato, M. A. F., Soares, W. V. B., Reis, C. B. M. (2010). Milk flow, teat morphology and subclinical mastitis prevalence in Gir cows. Pesquisa Agropecuária Brasileira, 45(12):1507-1512.

Porcionato, M.A.F., Negrão, J.A. \& Paiva, F.A. (2009). Morfometria e distribuição de leite alveolar e cisternal na glândula mamária de vacas Holandesa e Girolanda. Arquivo Brasileiro de Medicina Veterinaria e Zootecnia, 61 (2), 287-292.

Ramos, D. F., Silva, P. E. A., Dellagostin, O. A. (2015). Diagnosis of bovine tuberculosis: review of main techniques. Brazilian Journal Biology, 75(4):830-837.

Rathod, S. U., Khodwe, P. M., Raibole, R. D., Vyavahare, S. H. (2009). Theloscopy- the advancement in teat surgery and diagnosis. Veterinary World, 2:34-37.

Redelman, D., Butler, S., Robison, J., Garner, D. (1986). Identification of Inflammatory Cells in Bovine Milk by Flow Cytometry. Cytometric, 9:463-468.

Rekant, S. I., Lyons, M. A., Pacheco, J. M., Arzt, J., Rodriguez, L. L. (2016). Veterinary applications of infrared thermography. American Journal of Veterinary Research, 77(1):98-107.

Risvanli, A., Dogan, H., Safak, T., Kilic, M. A., Seker, I. (2019). The relationship between mastitis and the B-mode, colour Doppler ultrasonography measurements of supramammary lymph nodes in cows. Journal of Dairy Research, 86(3):315-318.

Rivas, A. L., Quimby, F. W., Blue, J., Coksaygan, O. (2001). Longitudinal evaluation of bovine mammary gland health status by somatic cell counting, flow cytometry, and cytology. Journal of Veterinary Diagnostic Investigation, 13:399-407.

Rizk, H. M., Khalifa, E. F., Abdelgalil, A. I. (2019). Comparative morphometric overview between the two milk systems of the mammary gland of one humped camel (Camelus dromedarius). RJPBCS, $8(3): 2417-2425$.

Rocha, A., Mineo, J. M., Lopes, E. R., Bessa, J. A. A., Ferreira, A. P., É Segadães Filho, A. J., Menezes, T. G E., Costa, M. C. (1987). O teste imunoenzimático ELISA no líquido pericárdico: novo método para o diagnóstico post-mortem da doença de chagas. Revista da Sociedade Brasileira de Medicina Tropical, 20(4):213-216.

Rodrigues, B. A., Rodrigues, J. L. (2009). Fatores associados com a acurácia da técnica ultrassonográfica no diagnóstico clínico em veterinária. A Hora Veterinária, 29:39-42.

Rodríguez, A. R., Olivares, F. J., Descouvieres, P. T., Werner, M. P., Tadich, N. A., Bustamante, H. A. (2016). Thermographic assessment of hoof temperature in dairy cows with different mobility scores. Livestock Science, 184:92-96.

Rothen, J., Pothier, J. F., Foucault, F., Blom, J., Nanayakkara, D., Li, C., Ip, M., Tanner, M., Vogel, G., Pflüger, V., Daubenberger, C. A. (2019). Subspecies Typing of Streptococcus agalactiae Based on Ribosomal Subunit Protein Mass Variation by MALDI-TOF MS. Frontiers of Microbiology, 10:1-13.

Sadan, M. (2019). Superficial swellings in sheep (Ovis aries) and goats (Capra hircus): Clinical and 1 ultrasonographic findings. The Journal of Veterinary Medical Science, 81(9):1326-1333.

Sadiq, M. B., Mansor, R., Syed-Hussain, S. S., Saharee, A. A., Zakaria, Z., Syahirah, A. A., Bousnane1, I., Jaezah Adlina, Z. A., Salleh, A., Wan Mohd Sukri, W. I., Mustaffa-Kamal, F., Ramanoon, S. Z. 
(2019). Clinical observation, acute phase protein levels, and histopathological changes of mammary gland in experimentally infected goats with Staphylococcus aureus. Comparative Clinical Pathology, 28(4):1069-1075.

Saleh, M. S., Mobarak, A. M., Foua, S. M. (1971). Radiological, Anatomical and histological Studies of the Mammary Gland of the One-humped Camel (Camelus dromedarius). Zbl. Veteterinary. Medicine Advances, 18:347-352.

Salles, M. S. V., Silva, S. C., Salles, F. A., Roma Jr, L. C., El Faro, L., Mac Lean, P. A. B., Oliveira, C. E. L., Martello, L. S. (2016). Mapping the body surface temperature of cattle by infrared thermography. Journal of Thermal Biology, 62:63-69.

Santos, V. J. C., Simplício, K. M. M. G., Sanchez, D. C. C., Almeida, V. T., Teixeira, P. P. M. (2014). Ultrassonografia convencional e doppler em cabra com mastite gangrenosa. Arquivo Brasileiro de Medicina Veterinária e Zootecnia, 66(6):1931-1935.

Santos, V. J. C., Simplicio, K., Sanchez, D., Coutinho, L., Teixeira, P., Barros, F., Almeida, V., Rodrigues, L., Bartlewski, P., Oliveira, M., Feliciano, M., Vicente, W. (2015). B-mode and doppler sonography of the mammary glands in dairy goats for mastitis diagnosis. Reproduction Domestics Animal, 50:251-255.

Saratsis P. \& Grunert E. (1993). Diagnostic ultrasound for the examination of the extension of teat stenosis or other anomalies of the teat in dairy cows. Deutsche Tierarztliche Wochenschrift, Hannover, $100,159-163$.

Sathiyabarathi, M., Jeyakumar, S., Manimaran, A., Pushpadass, H. A., Sivaram, M., Ramesha, K. P., Das, D. N., Kataktalware, M. A. (2018). Infrared thermal imaging of udder skin surface temperature variations to monitor udder health status in Bos indicus (Deoni) cows. Infrared Physics \& Technology, 88:239-244.

Sayed-Ahmed, A., Kulcsár, M., Rudas. P., Bartha, T. (2004). Expression and localisation of leptin and leptin receptor in the mammary gland of the dry and lactating non-pregnant cow. Acta Veterinaria Hungarica, 52 (1), 97-111.

Schwarz, D., Diesterbeck, U. S., König, S., Brügemann, K., Schlez, K., Zschöck, M., Wolter, W., Czerny, C. P. (2011). Flow cytometric differential cell counts in milk for the evaluation of inflammatory reactions in clinically healthy and subclinically infected bovine mammary glands. Journal of Dairy Science, 94:5033-5044.

Seeh, C., Hospes, R., Bostedt, H. (1996). The use of visual methods (sonography/endoscopy) for the diagnosis of a webbed teat in a cow. Tierarztliche Praxis, 24:438-42.

Shokry, M. M., Berbish, E. A. (2012). Case Report: Thelotomy in a Dairy Buffalo after Ultrasonographic Diagnosis of Teat Stenosis. Journal of Buffalo Science, 1:110-112.

Sinha, R., Bhakat, M., Mohanty, T. K., Ranjan, A., Kumar, R., Lone, S. A., Rahim, A., Paray, A. R., Khosla, K., Danish, Z. (2018). Infrared thermography as non-invasive technique for early detection of mastitis in dairy animals -A review. Asian Journal of Dairy \& Food Res, 37(1):1-6.

Sladek, Z., Rysanek, D. (2010). Apoptosis of resident and inflammatory macrophages before and during the inflammatory response of the virgin bovine mammary gland. Acta Veterinaria Scandinavica, 52(12):1-13.

Ślósarz, P., Wójtowski, J., Bielińska, S., Frąckowiak, A., Ludwiczak, A., Krzyŝewski, J., Bagnicka, E., Strzałkowska, N. (2010). Machine induced changes of caprine teats diagnosed by ultrasonography. African Journal of Biotechnology, 9(50):8698-8703.

Ślósarz, P., Wójtowski, J., Bielińska, S., Frąckowiak, A., Ludwiczak, A., Krzyżewski, J., Bagnicka, E., Strzalkowska, N. (2010). Machine induced changes of caprine teats diagnosed by ultrasonography. African Journal of Biotechnology, 9, 8698-8703.

Soler, L., García, N., Andrés, M., Armengol, R., Lampreave, F., Alava, M. A., Piñeiro, M. (2019). Development and validation of an ELISA for the quantification of bovine ITIH4 in serum and milk. Veterinary Immunology and Immunopathology, 217:1-6.

Sousa, F.C., Moraes, G. B., Viana, D. A., Rocha, D. D., Evangelista, J. S. A. M., Batista, R. I. T. P., Souza-Fabjan, J. M. G., Nunes-Pinheiro, D. C. S., Melo, L. M.,1 ,Freitas, V. J. F., Alves, D. I. (2015). Morphology and $\alpha$-SMA, F-actin and JC1 Protein Expression in the Mammary Gland of Goats in Early Hormonal Lactation. Acta Scientiae Veterinariae, 43:1301-1307. 
Takeda, T. (1989). Diagnostic ultrasound of the bovine udder. Japan Journal of Veterinary Research, $37: 133$.

Tavernor, W. D., Vaughan, L. C. (1962). Radiography of horses and cattle. British Veterinary Jounral, $118: 359-385$.

Thielen, M. A., Mielenz, M., Hiss, S., Zerbe, H., Petzl, W., Schuberth, H.J., Seyfert, H. M. \& Sauerwein, H. (2007). Short Communication: Cellular Localization of Haptoglobin mRNA in the Experimentally Infected Bovine Mammary Gland. Journal Dairy Science, 90, 1215-1219.

Thompson, K. G., Di Menna, M. E., Carter, M. E., Carman, M. G. (1978). Mycotic mastitis in two cows. New Zealand Veterinary Journal, 26(7):176-177.

Turner, T. A. (2001). Diagnostic thermography. Veterinary Clinics of North America: Equine Practice, 17(1):95-112.

Vangroenweghe, F., Broeck, W. V., Ketelaere, A., Bree, H. V., Duchateau, L; Burvenich, C. (2006). Endoscopic Examination and Tissue Sampling of the Bovine Teat and Udder Cistern, Journal of Dairy Science, 89:1516-1524.

Varvil, M. S., Haanen, G., Sola, M. F., Lin, T., Kritchevsky, J. E., Christian, J. A. (2019). A case of hypercalcemia in a heifer presumably due to mammary carcinoma bovine hypercalcemia of malignancy. Veterinary Clinical Pathology, 48:293-299.

Vianna, R. S., Batista, C. F., Gomes, R. C., Santos, K. R., Souza, F. N., Pogliani, F. C, Libera, A. M. M. P. D. (2017). Achados ultrassonográficos da glândula mamária de cabras naturalmente infectadas com o vírus da artrite encefalite caprina. Arquivo Brasileiro de Medicina Veterinária e Zootecnia, 69(1):65-74.

Vorm, O., Roepstorff, P., Mann, M. (1994). Improved Resolution and Very High Sensitivity in MALDI TOF of Matrix Surfaces Made by Fast Evaporation. Analytical. Chemical, 66:3281-3287.

Will, S., Wurgau S, T., Fraunholz, J. Bouadid, C., Leidl, W. (1990). Sonographic findings on the papilla mammae in cattle. Deutsche Tierarztliche Wochenschrift, 97, 403-406.

Winnicka, A., Klucinski, W., Hoser, G., Sikora, J., Kawiak, J. (1999). Flow Cytometry Analysis of Milk and Peripheral Blood Cells from Goats during Lactation. Journal of Veterinary and Medicine A, 46:459-464.

Winter, P., Colditz, I. G. (2002). Immunological responses of the lactating ovine udder following experimental challenge with Staphylococcus epidermidis. Veterinary Immunology and Immunopathology, 89:57-65.

Winter, P., Schilcher, F., Bago, Z., Schoder, D., Egerbacher, M., Baumgartner, W., Wagner, M. (2004). Clinical and Histopathological Aspects of Naturally Occurring Mastitis Caused by Listeria monocytogenes in Cattle and Ewes. Journal Veterinary and Medicine, 51:176-179.

Wójtowski, J., Ślósarz, P., Bielińska, S., Nowicki, S., Gut, A., Danków, R. (2006). Ultrasound image of morphological changes of teat end in sheep caused by machine milking. Archives Tierz. Dummerstorf, 49:231-237.

Worstorff, H., Steib, Jd., Prediger A., Schmidt, W. L. (1986). Assessment of ultrasonography for examination of teat changes in cows during milking. Milch Wissenschaft, 41:12-5.

Zhelavskyi, M. M., Dmytriv, O. Y., Mizyk, V. P. (2019). Changes in cellular factors of local immunity udder of cows with mastitis. Scientific Messenger of LNU of Veterinary Medicine and Biotechnologies. Series: Veterinary Sciences, 21(93):46-52.

Zoldan, K., Schneider, J., Moellmer, T., Fueldner, C., Knauer, J., Fuerll, M., Starke, A., Specht, M., Reiche, K., Hackermueller, J., Kalkhof, S., Von Bergen, M., Bergfeld, U., Fischer, R., Pache, S., Joerg, L. (2017). Discovery and Validation of Immunological Biomarkers in Milk for Health Monitoring of Dairy Cows - Results from a Multiomics Approach. Journal Advances Dairy Research, 5(3):1-19.

Recebido: 23 de novembro, 2019.

Aprovado: 24 de janeiro, 2020.

Publicado: 14 de maio, 2020.
Licenciamento: Este artigo é publicado na modalidade Acesso Aberto sob a licença Creative Commons Atribuição 4.0 (CC-BY 4.0), a qual permite uso irrestrito, distribuição, reprodução em qualquer meio, desde que o autor e a fonte sejam devidamente creditados. 